\title{
On the linear stability of nearly Kähler 6-manifolds
}

\author{
Uwe Semmelmann $^{1} \cdot$ Changliang Wang $^{2}$ (D) - M. Y.-K. Wang ${ }^{3}$
}

Received: 16 August 2019 / Accepted: 3 September 2019 / Published online: 13 September 2019

(c) The Author(s) 2019

\begin{abstract}
We show that a strict, nearly Kähler 6-manifold with either second or third Betti number nonzero is linearly unstable with respect to the $v$-entropy of Perelman and hence is dynamically unstable for the Ricci flow.
\end{abstract}

Keywords Linear stability $\cdot$ v-Entropy $\cdot$ Nearly Kähler 6-manifolds · Real Killing spinors

Mathematics Subject Classification 53C25 - 53C27 · 53C44

\section{Introduction}

Manifolds which admit a non-trivial Killing spinor form a distinguished subclass of Einstein manifolds. Recall that the Killing spinor equation is given by

$$
\nabla_{X} \sigma=c X \cdot \sigma
$$

where $\sigma$ is a complex spinor field, $c$ is a constant, $X$ is an arbitrary tangent vector, and . denotes Clifford multiplication. Let $(M, g)$ be the underlying Riemannian spin manifold and $n$ be its (real) dimension. Since a Killing spinor is an eigenspinor for the Dirac operator: $D \sigma=-n c \sigma$, the constant $c$ is zero (parallel spinor case), purely imaginary, or real.

In the $c=0$ case, we obtain special geometries of Calabi-Yau, hyperkähler, $\mathrm{G}_{2}$, and Spin(7) types. By the work of Dai et al. [9], the underlying Ricci-flat metric $g$ is linearly stable. When $c$ is purely imaginary, the manifolds were classified by Baum [2]. By the work of

\footnotetext{
$\triangle$ Changliang Wang

cwangmath@outlook.com

Uwe Semmelmann

uwe.semmelmann@mathematik.uni-stuttgart.de

M. Y.-K. Wang

wang@mcmaster.ca

1 Fachbereich Mathematik, Institut für Geometrie und Topologie, Universität Stuttgart, Pfaffenwaldring 57, 70569 Stuttgart, Germany

2 Max-Planck-Institut für Mathematik, Vivatsgasse 7, 53111 Bonn, Germany

3 Department of Mathematics and Statistics, McMaster University, Hamilton, ON L8S 4K1, Canada
} 
Kröncke [16] and the second author [24], the Einstein metrics (with negative scalar curvature) are also linearly stable.

When $c$ is real and nonzero, the Einstein metric $g$ has positive scalar curvature, and so by Lichnerowicz's theorem it cannot admit any harmonic spinors. Friedrich [12] then derived a positive lower bound for the eigenvalues of the square of the Dirac operator and furthermore showed that the lower bound is achieved precisely for those manifolds which admit a non-trivial Killing spinor. These manifolds are known to be locally irreducible and cannot be locally symmetric unless they are spherical space-forms (which we will exclude from our discussion henceforth). While they are far from being classified, there is a wellknown rough classification by Bär [1] in terms of the restricted holonomy of their metric cones $\left(\mathbb{R}_{+} \times M, d t^{2}+t^{2} g\right)$. The only possibilities are $\operatorname{SU}\left(\frac{n+1}{2}\right), \operatorname{Sp}\left(\frac{n+1}{4}\right), \mathrm{G}_{2}$, or $\operatorname{Spin}(7)$. Thus, $n$ can be even only if $n=6$, and, in this case, by the work of Grunewald [13] (see also chapter 5 in [3] $),(M, g)$ is either isometric to round $S^{6}$ or a strict nearly Kähler 6-manifold.

This article examines the linear stability of this class of Einstein 6-manifolds. Recall that a nearly Kähler manifold $(M, J, g)$ is an almost Hermitian manifold that satisfies

$$
\left(\nabla_{X} J\right) X=0
$$

for all tangent vectors $X$, where $\nabla$ denotes the Levi-Civita connection of $g$. The nearly Kähler structure is strict if it is not Kähler.

For the purpose of this paper, a closed Einstein manifold $(M, g)$ is linearly stable if for all transverse traceless (TT) symmetric 2-tensors $h$, i.e., divergence-free and trace-free symmetric 2-tensors, the quadratic form

$$
\mathcal{Q}(h, h)=-\left\langle\nabla^{*} \nabla h-2 \stackrel{R}{R} h, h\right\rangle_{L^{2}(M, g)} \leq 0 .
$$

In the above, $\stackrel{R}{R}$ is the action of the curvature tensor on symmetric 2-tensors (see (2.1)). $(M, g)$ is linearly unstable if it is not linearly stable. The coindex of a quadratic form is the dimension of the maximal subspace on which it is positive definite. More comments about stability will be given in Sect. 2. Here, we only mention that (a positive multiple of) the above quadratic form occurs in the second variation formula of both the Einstein-Hilbert action and Perelman's $v$-entropy.

The main result of this article is

Theorem 1.1 Let $(M, J, g)$ be a complete strict nearly Kähler 6-manifold. If $b_{2}(M)$ or $_{3}(M)$ is nonzero, then $g$ is linearly unstable with respect to the Einstein-Hilbert action restricted to the space of Riemannian metrics with constant scalar curvature and fixed volume. Hence, it is also linearly unstable with respect to the v-entropy of Perelman, and dynamically unstable with respect to the Ricci flow.

Note that an Einstein metric $g$ is dynamically unstable if there exists a non-trivial ancient rescaled Ricci flow $g_{t},-\infty<t \leq 0$, such that $g_{t}$ converges modulo diffeomorphisms to $g$ in the pointed Cheeger-Gromov topology. The conclusion about dynamic instability in the above theorem follows from Theorem 1.3 in [15].

The proof of Theorem 1.1 actually shows that the coindex of the Einstein metric $g$ (for either the Einstein-Hilbert action or $v$-functional) is $\geq b_{2}(M)+b_{3}(M)$.

By the theorem of Bonnet-Myers, a strict nearly Kähler 6-manifold has finite fundamental group. On the other hand, by pull-back any Riemannian cover of such a manifold also has a strict nearly Kähler structure. From the properties of the transfer homomorphism, the corresponding Betti numbers of any Riemannian cover are at least as large as those of the base. Hence, the nearly Kähler metrics on the covers are also linearly unstable. 
At present, there are very few examples of complete strict nearly Kähler 6-manifolds. Recently, Foscolo and Haskins produced the first non-homogeneous examples of such spaces [11]. One cohomogeneity one non-homogeneous nearly Kähler metric was produced on each of $S^{6}$ and $S^{3} \times S^{3}$. Our result implies that the second metric is dynamically unstable.

In [25], we showed that all the homogeneous nearly Kähler 6-manifolds other than the isotropy irreducible space $\mathrm{G}_{2} / \mathrm{SU}(3) \approx S^{6}$ are linearly unstable. Theorem 1.1 provides some additional information for these cases. In the case of $(\mathrm{SU}(2) \times \mathrm{SU}(2) \times \mathrm{SU}(2)) / \Delta \mathrm{SU}(2)$, it was shown in [25] that the eigenspace corresponding to the first nonzero eigenvalue of the Laplace-Beltrami operator of the nearly Kähler normal metric has dimension 12, and the corresponding eigenvalue is less than twice the Einstein constant. Hence, the normal metric is linearly unstable with respect to the $v$-entropy. However, the instability with respect to the Einstein-Hilbert action was unresolved. Theorem 1.1 shows that this is also the case, and further that the coindex of $g$ for the $v$-entropy is at least $12+2=14$. As for $\mathrm{Sp}(2) /(\mathrm{Sp}(1) \mathrm{U}(1))=\mathbb{C P}^{3}$, the Ziller metric was shown to be linearly unstable with respect to the Einstein-Hilbert action by appealing to the properties of its canonical variation as a Riemannian submersion type metric. The above theorem gives the instability without resorting to using fibrations or homogeneous geometry. Finally, the coindex of the nearly Kähler normal metric on $\mathrm{SU}(3) / T^{2}$ is at least 2 since the second Betti number is 2 in this case.

Finally, we mention that Theorem 1.1 can be interpreted as a rigidity result in the form of

Corollary 1.1 Let $(M, J, g)$ be a complete, simply connected, strict nearly Kähler manifold that is linearly stable with respect to the Einstein-Hilbert action. Then, it is a rational homology sphere. In particular, if $H_{2}(M, \mathbb{Z})$ has no torsion, then $M$ is diffeomorphic to $S^{6}$.

The corollary follows immediately from Theorem 1.1 by applying Wall's classification of closed simply connected spin 6-manifolds [23]. Recall that the absence of torsion in the second integral homology implies that there is no torsion in integral homology, and Wall showed that such manifolds are determined up to diffeomorphism by their integral homology type and their first Pontryagin class.

After recalling in the next section, the various notions of stability and those properties of nearly Kähler manifolds that will be used in this paper, the proof of Theorem 1.1 will be given in Sect. 3.

\section{Preliminaries and properties of nearly Kähler manifolds}

We begin with explicit statements of conventions used in this paper because different authors use different conventions for curvature quantities, and signs are of utmost importance for computations in the next sections. We take the $(1,3)$ curvature tensor to be $R_{X, Y}(Z)=$ $\left[\nabla_{X}, \nabla_{Y}\right] Z-\nabla_{[X, Y]} Z$. If $\left\{e_{1}, \ldots, e_{n}\right\}$ is an orthonormal frame, the $(0,4)$-curvature tensor is taken to be $R\left(e_{i}, e_{j}, e_{k}, e_{l}\right)=R_{i j k l}$. The sectional curvature determined by the 2-plane $\left\{e_{i}, e_{j}\right\}$ is $R_{i j j i}$. The action of the curvature on symmetric 2-tensors is given by

$$
(\stackrel{\circ}{R} h)_{i j}=-\sum_{p, q} R_{i p j q} h_{p q}
$$

Laplace-type operators will be consistent with the Laplace-Beltrami operator on functions given as $-\operatorname{tr}_{g}\left(\operatorname{Hess}_{g}\right)$, for which the eigenvalues are non-negative. 


\subsection{Notions of linear stability of Einstein metrics}

We next describe in more detail the various notions of stability mentioned in the Introduction. As is well known, Einstein metrics on closed manifolds are critical points of the total scalar curvature functional restricted to unit volume metrics. The second variation formula at an Einstein metric consists of three parts. For directions tangent to the orbit of the diffeomorphism group, the second variation is zero, and along directions corresponding to conformal changes, the second variation is non-negative as a consequence of the theorem of Lichnerowicz-Obata. Therefore, it is customary to associate linear stability of the Einstein-Hilbert functional with the second variation restricted to the space of transverse traceless symmetric 2-tensors (TTtensors), which is the tangent space of the space of unit volume constant scalar curvature metrics. By the work of Berger and Koiso, on this space the second variation is given by $\frac{1}{2} \mathcal{Q}(h, h)$, where $\mathcal{Q}$ is given by (1.1). Note that the operator $\nabla^{*} \nabla-2 R$ on TT-tensors at an Einstein metric with Einstein constant $\Lambda$ is the same as $\Delta_{L}-2 \Lambda \cdot \mathbb{I}$ where $\Delta_{L}$ is the Lichnerowicz Laplacian and $\mathbb{I}$ is the identity operator. The notion of linear instability given in the Introduction is equivalent to the condition $\left\langle\nabla^{*} \nabla h-2 \stackrel{R}{R} h, h\right\rangle_{L^{2}(M, g)}<0$ for some nonzero TT-tensor $h$.

Einstein metrics with positive scalar curvature also occur among the critical points of Perelman's $v$-entropy [20]. The second variation formula for this functional at an Einstein metric was computed by Cao et al. [6] and explained in detail in [7]. (For the corresponding formula at a shrinking gradient Ricci soliton, see [8].) It likewise consists of three parts. Along directions orthogonal to the orbit of the diffeomorphism group and along the space of TT-tensors, it agrees with that for the Einstein-Hilbert action (up to some positive constant factor). Along directions tangent to volume preserving conformal deformations, however, it can only have a positive definite subspace provided there are eigenfunctions of the LaplaceBeltrami operator with eigenvalues less than $2 \Lambda$. In other words, unstable directions are given by these eigenfunctions and by TT-tensors which are eigentensors of the Lichnerowicz Laplacian with eigenvalue $<2 \Lambda$.

Hence, Einstein metrics (with positive scalar curvature) which are linearly unstable with respect to the Einstein-Hilbert action are automatically linearly unstable with respect to the $v$-entropy. As mentioned in the Introduction, Kröncke's theorem implies that $v$-linearly unstable Einstein metrics are dynamically unstable with respect to the Ricci flow.

\subsection{Properties of nearly Kähler 6-manifolds}

As mentioned in the introduction, an almost Hermitian manifold $\left(M^{2 m}, g, J\right)$ is called nearly Kähler if $\left(\nabla_{X} J\right) X=0$ holds for all tangent vectors $X$, where $\nabla$ denotes the Levi-Civita connection of $g$. The canonical Hermitian connection $\bar{\nabla}$ is defined by $\bar{\nabla}_{X} Y=\nabla_{X} Y-$ $\frac{1}{2} J\left(\nabla_{X} J\right) Y$. This is a $\mathrm{U}(m)$ connection (i.e., $\bar{\nabla} g=0$ and $\bar{\nabla} J=0$ ) whose torsion $\bar{T}$, given by $\bar{T}_{X} Y:=-\frac{1}{2} J\left(\nabla_{X} J\right) Y$, is skew-symmetric. It is well known that for all nearly Kähler manifolds the torsion $\bar{T}$ is $\bar{\nabla}$-parallel. The Kähler form $\omega$ of $M$ is given by $\omega(\cdot, \cdot)=g(J \cdot, \cdot)$. The nearly Kähler condition implies that the tensor $\Psi^{+}:=\nabla \omega$ is a 3-form.

Recall that the almost complex structure $J$ acts as an automorphism on the space of complex-valued differential forms and induces a (pointwise) orthogonal decomposition of this space into forms of type $(p, q)$. Our convention here is that of [4], so that $J$ acts on a form of type $(p, q)$ as multiplication by $i^{q-p}$. We denote by $\Lambda^{(p, q)+(q, p)} \mathrm{T} M$ the space of forms of type $(p, q)+(q, p)$, i.e., the projection of the complex bundle $\Lambda^{(p, q)} \mathrm{T} M$ onto the real bundle $\Lambda^{p+q} \mathrm{~T} M$, e.g., the Kähler form $\omega$ is of type $(1,1)$ and $\Psi^{+}$is of type $(3,0)+(0,3)$. 
Similarly, the bundle SymT $M$ of $g$-symmetric endomorphisms splits into the orthogonal direct sum SymT $M=\mathrm{Sym}^{+} \mathrm{T} M \oplus \mathrm{Sym}^{-} \mathrm{T} M$, of symmetric endomorphisms commuting, respectively, anti-commuting, with the almost complex structure $J$. The trace of every element in $\mathrm{Sym}^{-} \mathrm{T} M$ is automatically 0 , and $\mathrm{Sym}^{+} \mathrm{T} M$ decomposes further as $\operatorname{Sym}^{+} \mathrm{T} M=$ $\mathrm{Sym}_{0}^{+} \mathrm{T} M \oplus \mathbb{I}$, i.e., into its trace-free part and multiples of the identity.

From now on we assume that $M$ is 6-dimensional and that the nearly Kähler manifold is strict, i.e., $(M, g, J)$ is not Kähler. In this situation, the metric $g$ is Einstein and we assume its scalar curvature is normalized to be 30 . It turns out that the 3 -form $\Psi^{+}$has pointwise constant norm and can thus be considered as the real part of a $\bar{\nabla}$-parallel complex volume form $\Psi=\Psi^{+}+i \Psi^{-}$, where $\Psi^{-}=* \Psi^{+}$. Thus, $M$ carries a SU(3)-structure with minimal connection $\bar{\nabla}$, which has holonomy contained in $\mathrm{SU}(3)$.

We will need identifications of the subbundles $\operatorname{Sym}^{ \pm} \mathrm{T} M$ with spaces of forms. First, the bundle $\operatorname{Sym}_{0}^{+} \mathrm{T} M$ can be identified with $\Lambda_{0}^{(1,1)} \mathrm{T} M$, the bundle of primitive $J$-invariant 2 -forms. The isomorphism is given by the map $h \mapsto \eta=h(J \cdot, \cdot)$. Moreover, the bundle Sym $^{-} \mathrm{T} M$ can be identified with the bundle $\Lambda_{0}^{(2,1)+(1,2)} \mathrm{T} M$ of primitive 3-forms of type $(2,1)+(1,2)$. Here, the isomorphism is given by the map $h \mapsto h_{*} \Psi^{+}$, where $h_{*}$ denotes the natural action of endomorphisms on forms defined by $\left.h_{*} \Psi^{+}=-\sum_{i} h\left(e_{i}\right) \wedge e_{i}\right\lrcorner \Psi^{+}$, for any orthonormal basis $\left\{e_{i}\right\}$ of $T$. For more details, we refer to [17], p. 60. These two identifications are $\bar{\nabla}$-parallel bundle isomorphisms since they are fibrewise defined by SU(3)-equivariant maps.

In the following, it will be convenient to consider the standard Laplacian $\Delta_{V}$ introduced in [21]. It is a Laplace-type operator acting on sections of any vector bundle $V M$ associated with the (oriented orthonormal) frame bundle $P$ via a representation $(V, \rho)$ of $\mathrm{SO}(n)$. It is defined as $\Delta_{V}=\nabla^{*} \nabla+q(R)$, where $\nabla$ is the covariant derivative induced by the LeviCivita connection and $q(R)$ is a symmetric endomorphism of $V M$, linear in the Riemannian curvature $R$ and defined fibrewise by $q(R)=\frac{1}{2} \sum_{i, j}\left(e_{i} \wedge e_{j}\right)_{*} R\left(e_{i} \wedge e_{j}\right)_{*}$. For any 2-form $A \in \Lambda^{2} \mathbb{R}^{n} \cong \mathfrak{s o}(n)$, we denote by $A_{*}$ the action of $A$ on $V$ via the differential of $\rho$.

The motivating example is the standard form representation $V=\Lambda^{k} \mathrm{~T}$. Then, $\Delta_{V}$ is the Hodge-Laplace operator $\Delta=d d^{*}+d^{*} d$ on differential forms. Another important example is $V=$ SymT. Here, the standard Laplace operator coincides with the Lichnerowicz Laplacian $\Delta_{L}$ on symmetric endomorphisms, or equivalently symmetric 2-tensors. In particular, we have $q(R)=-2 \stackrel{\circ}{R}+\operatorname{Ric}$, where $(\operatorname{Ric} h)(X, Y):=h(\operatorname{Ric} X, Y)+h(X, \operatorname{Ric} Y)$ denotes the standard derivative extension of the Ricci endomorphism to symmetric 2-tensors and the curvature operator $\stackrel{\circ}{R}$ is defined by $(2.1)$, or equivalently by $(\stackrel{\circ}{R} h)(X, Y)=-\sum_{i} h\left(R_{X, e_{i}} Y, e_{i}\right)$ (cf. [21], p. 280).

More generally, one can consider a Laplace-type operator $\bar{\Delta}_{V}:=\bar{\nabla}^{*} \bar{\nabla}+q(\bar{R})$, where instead of the Levi-Civita connection $\nabla$ one has a metric connection $\bar{\nabla}$ with curvature $\bar{R}$. The frame bundle $P$ has to be replaced by a $G$-principal bundle with $G=\operatorname{Hol}(\bar{\nabla})$. Here, $V$ is a $G$-representation and it is reasonable to consider only connections with skew-symmetric and parallel torsion. In particular, if $\bar{\nabla}$ is the canonical hermitian connection of a nearly Kähler manifold, the standard Laplace operator $\bar{\Delta}_{V}$ is exactly the Hermitian Laplacian $\bar{\Delta}$ introduced in [19], p. 254. An important property of the standard Laplacian is that it commutes with all parallel bundle maps (cf. [21], p. 283).

Regarding harmonic forms on $M$ we shall need to use the following result of Verbitsky:

Theorem 2.1 ([22], Theorem 6.2) Let ( $M, J, g)$ be a strict nearly Kähler 6-manifold. Then, the space of harmonic $k$-forms is a direct sum of spaces $\mathcal{H}^{p, q}$ of harmonic forms of type $(p, q)$ with $k=p+q$, and $\mathcal{H}^{p, q}=0$ unless $p=q$ or $(p, q)=(2,1)$ or $(1,2)$. All harmonic $(1,1)$-forms are primitive, as are all harmonic 3-forms. 
An alternative proof of the above result can be found on p. 598 of [10].

\section{Proof of Theorem 1.1}

\subsection{The $b_{2}(M) \neq 0$ case}

Recall that Cao, Hamilton, and Illmanen observed in [6], pp. 6-7, that a compact shrinking Kähler Ricci soliton with $b^{1,1} \geq 2$ is linearly unstable and gave a simple argument for KählerEinstein case. (For the case of Kähler Ricci solitons, see [14]). Our result may be viewed as the analog of this observation for complete, strict, nearly Kähler 6-manifolds. In this case, the fundamental 2-form $\omega$ is not closed, and by Verbitsky's theorem, any harmonic 2-form is pointwise orthogonal to $\omega$. Hence, the analogous condition is $b_{2}(M)>0$ instead.

Let $\eta$ be a harmonic 2-form and $h(X, Y):=\eta(J X, Y)$. By Verbitsky's theorem, $\eta$ is $J$-invariant and primitive. So $h$ is a $J$-invariant symmetric 2-tensor. Since $\eta$ is pointwise orthogonal to $\omega$, it follows that $\operatorname{tr}_{g} h=0$. The harmonic form $\eta$ is closed and co-closed, which implies by a short calculation that $h$ is divergence free (cf. Lemma 4.2 in [17]).

As a consequence of Proposition 3.4 and Corollary 4.4 in [18], we have that $\Delta$ and $\bar{\Delta}$ coincide on primitive co-closed $(1,1)$-forms. Hence, $\bar{\Delta} \eta=\Delta \eta=0$. Since the standard Laplace operator $\bar{\Delta}$ commutes with $\bar{\nabla}$-parallel bundle homomorphims, we also have $\bar{\Delta} h=0$. Using the definition of $\bar{\Delta}$ and again Proposition 3.4 and Corollary 4.4 of [18] we obtain

$$
0=\bar{\nabla}^{*} \bar{\nabla} h+q(\bar{R}) h=\nabla^{*} \nabla h-3 h+q(R) h-3 h=\nabla^{*} \nabla h+q(R) h-6 h .
$$

Since the nearly Kähler metric $g$ is Einstein with scalar curvature normalized to be 30 , it follows that Ric $h=10 h$. Hence,

$$
\nabla^{*} \nabla h-2 \stackrel{\circ}{R} h=\nabla^{*} \nabla h+q(R) h-10 h=-4 h .
$$

\subsection{The $b_{3}(M) \neq 0$ case}

We construct a destabilizing TT symmetric 2-tensor from any given harmonic 3-form as follows. Let $\eta$ be a harmonic 3-form. Then, by Verbitsky's theorem, it is primitive and of type $(2,1)+(1,2)$. Hence, as recalled above, there is a symmetric endomorphism $h \in \mathrm{Sym}^{-} \mathrm{T} M$ such that $\eta=h_{*} \Psi^{+}$. It follows that $h$ is divergence free (Lemma 4.2, (35) in [17]). Indeed, $h_{*} \Psi^{-}=-*\left(h_{*} \Psi^{+}\right)$(cf. [17], p. 61). Thus, $d\left(h_{*} \Psi^{-}\right)=-d *\left(h_{*} \Psi^{+}\right)=* d^{*} \eta=0$, since $\eta$ is co-closed as a harmonic form. Again a combination of Proposition 3.4 and Corollary 4.4 in [18] shows that $\Delta$ and $\bar{\Delta}$ coincides on co-closed 3-forms in $\Lambda^{(2,1)+(1,2)} \mathrm{T} M$. Hence, $\bar{\Delta} \eta=\Delta \eta=0$ and as above we can conclude that $\bar{\Delta} h=0$. This can be reformulated as

$$
0=\bar{\nabla}^{*} \bar{\nabla} h+q(\bar{R}) h=\nabla^{*} \nabla h-2 h+q(R) h-2 h=\nabla^{*} \nabla h+q(R) h-4 h,
$$

by using again Proposition 3.4 and Corollary 4.4 of [18]. In this case, it follows that

$$
\nabla^{*} \nabla h-2 \stackrel{\circ}{R} h=\nabla^{*} \nabla h+q(R) h-10 h=-6 h .
$$

This completes the proof of Theorem 1.1. 
Remark 3.1 As one can easily check by using SU(3)-equivariance, an explicit expression of the destabilizing direction $h$ in terms of $\eta$ is given by

$$
\begin{aligned}
h(X, Y) & =-\frac{1}{8} \sum_{i, j}\left(\eta\left(X, e_{i}, e_{j}\right) \Psi^{+}\left(Y, e_{i}, e_{j}\right)+\eta\left(Y, e_{i}, e_{j}\right) \Psi^{+}\left(X, e_{i}, e_{j}\right)\right) \\
& =-\frac{1}{4}\left(\left\langle i_{X} \eta, i_{Y} \Psi^{+}\right\rangle+\left\langle i_{Y} \eta, i_{X} \Psi^{+}\right\rangle\right),
\end{aligned}
$$

where $\langle$,$\rangle denotes the inner product for 2-forms. Indeed, the righthand side is the inverse$ of the isomorphism $h \mapsto \eta=h_{*} \Psi^{+}$mentioned above. The above formula is the analog of Bryant's formula (2.17) in [5] for the nearly parallel $G_{2}$ case.

Acknowledgements Open access funding provided by Max Planck Society. C. Wang gratefully acknowledges the support and wonderful working condition of the Max Planck Institute for Mathematics in Bonn. M. Wang's research is partially supported by NSERC Grant No. OPG0009421. Both of them like to thank Xianzhe Dai, Stuart Hall, Fei He, J. Madnick, Tommy Murphy, and Guofang Wei for discussions and comments on an earlier version of the paper.

Open Access This article is distributed under the terms of the Creative Commons Attribution 4.0 International License (http://creativecommons.org/licenses/by/4.0/), which permits unrestricted use, distribution, and reproduction in any medium, provided you give appropriate credit to the original author(s) and the source, provide a link to the Creative Commons license, and indicate if changes were made.

\section{References}

1. Bär, C.: Real Killing spinors and holonomy. Comm. Math. Phys. 154, 509-521 (1993)

2. Baum, H.: Complete Riemannian manifolds with imaginary Killing spinors. Ann. Glob. Anal. Geom. 7(3), 205-226 (1989)

3. Baum, H., Friedrich, T., Grunewald, R., Kath. I.: Twistors and Killing Spinors on Riemannian Manifolds, Teubner-Texte zur Mathematik, Band, vol. 124 (1991)

4. Besse, A.L.: Einstein Manifolds. Springer, Berlin (1987)

5. Bryant, R.: Some remarks on $G_{2}$-structures. In: Proceedings of Gökova Geometry Topology Conference, vol. 2006, pp. 75-109 (2005)

6. Cao, H.-D., Hamilton, R., Ilmanen, T.: Gaussian Densities and Stability for Some Ricci Solitons. arXiv:math/0404165 (2004)

7. Cao, H.-D., He, C.: Linear stability of Perelman's v-entropy on symmetric spaces of compact type. J. Reine Angew. Math. 709, 229-246 (2015)

8. Cao, H.-D., Zhu, M.: On second variation of Perelman's Ricci shrinker entropy. Math. Ann. 353, 747-762 (2012)

9. Dai, X., Wang, X., Wei, G.: On the stability of Riemannian manifold with parallel spinors. Invent. Math. 161(1), 151-176 (2005)

10. Foscolo, R.: Deformation theory of nearly Kähler manifolds. J. Lond. Math. Soc. 95, 586-612 (2017)

11. Foscolo, F., Haskins, M.: New $\mathrm{G}_{2}$-holonomy cones and exotic nearly Kähler structures on $S^{6}$ and $S^{3} \times S^{3}$. Ann. Math. 185, 59-130 (2017)

12. Friedrich, T.: Die erste Eigenwert des Dirac-operators einer kompakten Riemannschen Mannigfaltigkeit nichtnegativer Skalarkrümmung. Math. Nach. 97, 117-146 (1980)

13. Grunewald, R.: Six-dimensional Riemannian manifolds with a real Killing spinor. Ann. Glob. Anal. Geom. 8, 43-59 (1990)

14. Hall, S.T., Murphy, T.: On the linear stability of Kähler-Ricci solitons. Proc. Amer. Math. Soc. 139(9), 3327-3337 (2011)

15. Kröncke, K.: Stability and instability of Ricci solitons. Calc. Var. PDE 53, 265-287 (2015)

16. Kröncke, K.: Stable and unstable Einstein warped products. Trans. Amer. Math. Soc. 365(9), 6537-6563 (2017)

17. Moroianu, A., Nagy, P.-A., Semmelmann, U.: Deformation of nearly Kähler structures. Pac. J. Math. 235, 57-72 (2008) 
18. Moroianu, A., Semmelmann, U.: Infinitesimal Einstein deformations of nearly Kähler metrics. Trans. Amer. Math. Soc. 363(6), 3057-3069 (2011)

19. Moroianu, A., Semmelmann, U.: The Hermitian Laplace operator on nearly Kähler manifolds. Comm. Math. Phys. 294, 251-272 (2010)

20. Perelman, G.: The Entropy Formula for the Ricci Flow and Its Geometric Applications. arXiv:math/0211159 (2002)

21. Semmelmann, U., Weingart, G.: The standard Laplace operator. Manuscripta Math. 158(1-2), 273-293 (2019)

22. Verbitsky, M.: Hodge theory on nearly Kähler manifolds. Geom. Topol. 15, 2111-2133 (2011)

23. Wall, C.T.C.: On certain 6-manifolds. Invent. Math. 1, 355-374 (1966)

24. Wang, C.: Stability of Riemannian manifolds with Killing spinors. Int. J. Math. 28(1), 1750005 (2017). 19 pages

25. Wang, C., Wang, Y. K.: Instability of Some Riemannian Manifolds with Real Killing Spinors. arXiv: 1810.04526

Publisher's Note Springer Nature remains neutral with regard to jurisdictional claims in published maps and institutional affiliations. 